\title{
Hopf-Pitchfork Bifurcation in a Phytoplankton-Zooplankton Model with Delays
}

\author{
Jia-Fang Zhang and Dan Zhang \\ School of Mathematics and Information Sciences, Henan University, Kaifeng 475001, China \\ Correspondence should be addressed to Jia-Fang Zhang; jfzhang@henu.edu.cn
}

Received 21 October 2013; Accepted 12 November 2013

Academic Editor: Allan Peterson

Copyright ( 2013 J.-F. Zhang and D. Zhang. This is an open access article distributed under the Creative Commons Attribution License, which permits unrestricted use, distribution, and reproduction in any medium, provided the original work is properly cited.

The purpose of this paper is to study the dynamics of a phytoplankton-zooplankton model with toxin delay. By studying the distribution of the eigenvalues of the associated characteristic equation, the pitchfork bifurcation curve of the system is obtained. Furthermore, on the pitchfork bifurcation curve, we find that the system can undergo a Hopf bifurcation at the positive equilibrium, and we derive the critical values where Hopf-Pitchfork bifurcation occurs.

\section{Introduction}

The study of the dynamical interaction of zooplankton and phytoplankton is an important area of research in marine ecology. Phytoplanktons are tiny floating plants that live near the surface of lakes and ocean. They provide food for marine life, oxygen for human being, and also absorb half of the carbon dioxide which may be contributing to the global warming [1]. Zooplanktons are microscopic animals that eat other phytoplankton. Toxins are produced by phytoplankton to avoid predation by zooplankton. The toxin producing phytoplankton not only reduces the grazing pressure on them but also can control the occurrence of bloom; see Chattopadhyay et al. [2] and Sarkar and Chattopadhyay [3]. Phytoplankton-zooplankton models have been studied by many authors [4-9]. In [6], models of nutrient-plankton interaction with a toxic substance that inhibit either the growth rate of phytoplankton, zooplankton, or both trophic levels are proposed and studied. In [7], authors have dealt with a nutrient-plankton model in an aquatic environment in the context of phytoplankton bloom. Roy [8] has constructed a mathematical model for describing the interaction between a nontoxic and a toxic phytoplankton under a single nutrient. Saha and Bandyopadhyay [9] considered a toxin producing phytoplankton-zooplankton model in which the toxin liberation by phytoplankton species follows a discrete time variation. Biological delay systems of one type or another have been considered by a number of authors [10, 11]. These systems governed by integrodifferential equations exhibit much more rich dynamics than ordinary differential systems. For example, Das and Ray [5] investigated the effect of delay on nutrient cycling in phytoplankton-zooplankton interactions in the estuarine system. In this paper we present a phytoplankton-zooplankton model to investigate its dynamic behaviors. The model we considered is based on the following plausible toxic-phytoplankton-zooplankton systems introduced by Chattopadhayay et al. [2]

$$
\begin{gathered}
\frac{d P}{d t}=r_{1} P\left(1-\frac{P}{K}\right)-a P Z, \\
\frac{d Z}{d t}=b Z \int_{-\infty}^{t} G(t-s) P(s) d s-c Z-d \frac{P(t-\tau)}{e+P(t-\tau)} Z,
\end{gathered}
$$

where $P(t)$ and $Z(t)$ are the densities of phytoplankton and zooplankton, respectively. $r_{1}, K, a, b, c, d$, and $e$ are positive constants. $\tau$ is toxin delay, $G(s)$ is the delay kernel and a nonnegative bounded function defined on $[0, \infty]$ as follows:

$$
\int_{0}^{\infty} G(s) d s=1, \quad G(s)=\sigma e^{-\sigma s}, \quad \sigma>0 .
$$

For a set of different species interacting with each other in ecological community, perhaps the simplest and probably the most important question from a practical point of view is 
whether all the species in the system survive in the long term. Therefore, the periodic phenomena of biological system are often discussed [12-16].

The primary purpose of this paper is to study the effects of toxin delay on the dynamics of (1). That is to say, we will take the delay $\tau$ passes through a critical value, the positive equilibrium loses its stability and bifurcation occurs. By studying the distribution of the eigenvalues of the associated characteristic equation, the pitchfork bifurcation curve of the system is obtained. Furthermore, we derive the critical values where Hopf-Pitchfork bifurcation occurs.

The paper is structured as follows. In Section 2, we discuss the local stability of the positive solutions and the existence of Pitchfork bifurcation. In Section 3, the conditions for the occurrence of Hopf-Pitchfork bifurcation are determined.

\section{Stability and Pitchfork Bifurcation}

In this section, we focus on investigating the local stability and the existence of Pitchfork bifurcation of the positive equilibrium of system (1). It is easy to see that system (1) has a unique positive equilibrium $E^{*}\left(P^{*}, Z^{*}\right)$, where

$$
\begin{gathered}
P^{*}=\frac{c+d-b e+\sqrt{(c+d-b e)^{2}+4 b c e}}{2 b}, \\
Z^{*}=\frac{r}{a}\left(1-\frac{P^{*}}{K}\right)>0
\end{gathered}
$$

where $\left(H_{1}\right): K>P^{*}$.

Let

$$
W(t)=\int_{-\infty}^{t} \sigma e^{-\sigma(t-s)} P(s) d s .
$$

By the linear chain trick technique, then system (1) can be transformed into the following system:

$$
\begin{gathered}
\frac{d P}{d t}=r_{1} P\left(1-\frac{P}{K}\right)-a P Z, \\
\frac{d Z}{d t}=b Z W-c Z-d \frac{P(t-\tau)}{e+P(t-\tau)} Z, \\
\frac{d W}{d t}=\sigma P(t)-\sigma W(t) .
\end{gathered}
$$

It is easy to check that system (5) has an unique positive equilibrium $E\left(P^{*}, Z^{*}, W^{*}\right)$ with $P^{*}=W^{*}$ provided that the condition $\left(H_{1}\right)$ holds.

Let $P=u+u^{*}, Z=v+v^{*}$, and $W=w+W^{*}$; then system (5) can be transformed into the following system:

$$
\begin{gathered}
\dot{u}(t)=-\frac{r}{K} P^{*} u(t)-a P^{*} v(t)-a u(t) v(t)-\frac{r}{K} u^{2}(t) \\
\dot{v}(t)=-\frac{d e}{\left(e+P^{*}\right)} Z^{*} u(t-\tau)+b Z^{*} w(t) \\
+\sum_{i+j+k \geq 2} f_{2}^{(i j k)} u^{i}(t-\tau) v^{j} w^{k} \\
\dot{w}(t)=\sigma u(t)-\sigma w(t)
\end{gathered}
$$

where

$$
\begin{aligned}
& f_{2}^{(i j k)}=\frac{1}{i ! j ! k !} \frac{\partial^{i+j+k} f_{2}}{\partial u^{i}(t-\tau) \partial v^{j} \partial w^{k}}, \\
& f_{2}=b v w-c v-d \frac{u(t-\tau)}{e+u(t-\tau)} v .
\end{aligned}
$$

Then linearizing system $(6)$ at $E^{*}\left(P^{*}, Z^{*}, W^{*}\right)$ is

$$
\begin{gathered}
\dot{u}(t)=-\frac{r}{K} P^{*} u(t)-a P^{*} v(t), \\
\dot{v}(t)=-\frac{d e}{\left(e+P^{*}\right)} Z^{*} u(t-\tau)+b Z^{*} w(t), \\
\dot{w}(t)=\sigma u(t)-\sigma w(t) .
\end{gathered}
$$

It is easy to see that the associated characteristic equation of system (11) at the positive equilibrium has the following form and thus the characteristic equation of system (5) is given by

$$
F(\lambda)=\lambda^{3}+p_{2} \lambda^{2}+p_{1} \lambda+p_{0}-\left[q_{1} \lambda+q_{0}\right] e^{-\lambda \tau}=0,
$$

where

$$
\begin{gathered}
p_{2}=\sigma+\frac{r P^{*}}{K}, \quad p_{1}=\frac{r P^{*} \sigma}{K}, \quad p_{0}=a b P^{*} Z^{*} \sigma, \\
q_{1}=\frac{a d e P^{*} Z^{*}}{\left(e+P^{*}\right)^{2}}, \quad q_{0}=\frac{a d e P^{*} Z^{*} \sigma}{\left(e+P^{*}\right)^{2}} .
\end{gathered}
$$

Obviously, $p_{2}>0, p_{1}>0, p_{0}>0, q_{1}>0$, and $q_{0}>0$.

From (9), the following lemma is obvious.

Lemma 1. If the condition $H_{2}: p_{0}=q_{0}$ holds, then $\lambda=0$ is always a root of (9) for all $\tau \geq 0$.

Let $d_{0}=(b / e)\left(e+P^{*}\right)^{2}, d_{1}=p_{1}\left(e+P^{*}\right)^{2} /(1-\tau \sigma) a e P^{*} Z^{*}$, and $d_{2}=\left(e+P^{*}\right)^{2}\left[\tau^{2} a b P^{*} Z^{*} \sigma-2\left(\sigma+\left(r P^{*} / K\right)\right)\right] / 2 \tau a e P^{*} Z^{*}$; we have the following results.

Lemma 2. Suppose that the condition $\left(\mathrm{H}_{2}\right)$ is satisfied.

(i) If $d=d_{0} \neq d_{1}$, then (9) has a single zero root.

(ii) If $d=d_{1} \neq d_{2}$, then (9) has a double zero root.

Proof. Clearly, $\lambda=0$ is a root to (9) if and only if $p_{0}=q_{0}$, which means $d=d_{0}$. Substituting $d=d_{0}$ into $F(\lambda)$ and taking the derivative with respect to $\lambda$, we obtain

$$
\left.F^{\prime}(\lambda)\right|_{d=d_{0}}=3 \lambda^{2}+2 p_{2} \lambda+p_{1}-\left[q_{1}(1-\tau \lambda)-\tau q_{0}\right] e^{-\lambda \tau} .
$$

Then we can get

$$
\left.F^{\prime}(0)\right|_{d=d_{0}}=p_{1}-\left[q_{1}-\tau q_{0}\right]
$$

For any $\tau>0$, by solving (12), we can obtain $d=d_{1}$. If $d=$ $d_{0} \neq d_{1}, F^{\prime}(0) \neq 0$ which means that $\lambda=0$ is a single zero root to (9), and hence the conclusion of (i) follows. 
From (11), it follows that

$$
\left.F^{\prime \prime}(\lambda)\right|_{d=d_{1}}=6 \lambda+2 p_{2}-\left[q_{1}\left(-2 \tau+\tau^{2} \lambda\right)+\tau^{2} q_{0}\right] e^{-\lambda \tau} .
$$

Then we get

$$
\left.F^{\prime \prime}(0)\right|_{d=d_{1}}=2 p_{2}-\left[\tau^{2} q_{0}-2 q_{1} \tau\right] .
$$

For any $\tau>0$, by solving (14), we can obtain $d=d_{2}$. If $d=d_{1} \neq d_{2}, F^{\prime \prime}(0) \neq 0$ which means that $\lambda=0$ is a double zero root to (9), and hence the conclusion of (ii) follows. This completes the proof.

From Lemma 2, we have the following result.

Theorem 3. Suppose that $\left(H_{2}\right)$ holds if $d=d_{0} \neq d_{1}$, then, the system (5) undergoes a Pitchfork bifurcation at the positive equilibrium.

\section{Hopf-Pitchfork Bifurcation}

In the following, we consider the case that (9) not only has a zero root, but also has a pair of purely imaginary roots $\pm i \omega$ $(\omega>0)$, when $d=d_{0} \neq d_{1}$ holds.

Substituting $\lambda=i \omega(\omega>0)$ and $d=d_{0}$ into (9) and separating the real and imaginary parts, one can get

$$
\begin{aligned}
& -\omega^{3}+p_{1} \omega-q_{1} \omega \cos (\omega \tau)+q_{0} \sin (\omega \tau)=0, \\
& -p_{2} \omega^{2}+p_{0}-q_{1} \omega \sin (\omega \tau)-q_{0} \cos (\omega \tau)=0 .
\end{aligned}
$$

It is easy to see from (15) that

$$
\omega^{6}+D_{2} \omega^{4}+D_{1} \omega^{2}+D_{0}=0
$$

where

$$
\begin{gathered}
D_{2}=p_{2}^{2}-2 p_{1}, \quad D_{1}=p_{1}^{2}-2 p_{0} p_{2}-q_{1}^{2}, \\
D_{0}=p_{0}^{2}-q_{0}^{2} .
\end{gathered}
$$

Let $z=\omega^{2}$. Then (16) can be written as

$$
h(z)=z^{3}+D_{2} z^{2}+D_{1} z+D_{0} .
$$

In terms of the coefficient in $h(z)$ define $\Delta$ by $\Delta=D_{2}^{2}-3 D_{1}$. It is easy to know from the characters of cubic algebraic equation that $h(z)$ is a strictly monotonically increasing function if $\Delta \leq 0$. If $\Delta>0$ and $z^{*}=\left(\sqrt{\Delta}-D_{2}\right) / 3<0$ or $\Delta>0$, $z^{*}=\left(\sqrt{\Delta}-D_{2}\right) / 3>0$ but $h\left(z^{*}\right)>0$, then $h(z)$ has always no positive root. Therefore, under these conditions, (9) has no purely imaginary roots for any $\tau>0$ and this also implies that the positive equilibrium $E\left(P^{*}, Z^{*}, W^{*}\right)$ of system (1) is absolutely stable. Thus, we can obtain easily the following result on the stability of positive equilibrium $E\left(P^{*}, Z^{*}, W^{*}\right)$ of system (1).

Theorem 4. Assume that $\left(H_{1}\right)$ holds and $\Delta \leq 0$ or $\Delta>0$ and $z^{*}=\left(\sqrt{\Delta}-D_{2}\right) / 3<0$ or $\Delta>0, z^{*}>0$ and $h\left(z^{*}\right)>$ 0 . Then the positive equilibrium $E\left(P^{*}, Z^{*}, W^{*}\right)$ of system (5) is absolutely stable; namely; $E\left(P^{*}, Z^{*}, W^{*}\right)$ is asymptotically stable for any delay $\tau \geq 0$.
In what follows, we assume that the coefficients in $h(z)$ satisfy the condition

$$
\left(H_{3}\right) \Delta=D_{2}^{2}-3 D_{1}>0, z^{*}=\left(\sqrt{\Delta}-D_{2}\right) / 3>0, h\left(z^{*}\right)<0 .
$$

Then, according to Lemma 2.2 in [17], we know that (16) has at least a positive root $\omega_{0}$; that is, the characteristic equation (9) has a pair of purely imaginary roots $\pm i \omega_{0}$. Eliminating $\sin (\omega \tau)$ in (15), we can get that the corresponding $\tau_{k}>0$ such that (9) has a pair of purely imaginary roots $\pm i \omega_{0}, \tau_{k}>0$ are given by

$$
\begin{aligned}
\tau_{k}= & \frac{1}{\omega_{0}} \arccos \left[\frac{-q_{1} \omega_{0}^{4}+\left(p_{1} q_{1}-p_{2} q_{0}\right) \omega_{0}^{2}+p_{0} q_{0}}{q_{1}^{2} \omega_{0}^{2}+q_{0}^{2}}\right] \\
& +\frac{2 k \pi}{\omega_{0}}, \quad(k=0,1,2, \ldots) .
\end{aligned}
$$

Let $\lambda(\tau)=v(\tau)+i \omega(\tau)$ be the roots of (9) such that when $\tau=\tau_{k}$ satisfying $v\left(\tau_{k}\right)=0$ and $\omega\left(\tau_{k}\right)=\omega_{0}$. We can claim that

$$
\operatorname{sgn}\left[\frac{d(\operatorname{Re} \lambda)}{d \tau}\right]_{\tau=\tau_{k}}=\operatorname{sgn}\left\{h^{\prime}\left(\omega_{0}^{2}\right)\right\} .
$$

In fact, differentiating two sides of (9) with respect to $\tau$, we get

$$
\begin{aligned}
\left(\frac{d \lambda}{d \tau}\right)^{-1} & =-\frac{\left(3 \lambda^{2}+2 p_{2} \lambda+p_{1}\right)-q_{1} e^{-\lambda \tau}+\left(q_{1} \lambda+q_{0}\right) \tau e^{-\lambda \tau}}{\left(q_{1} \lambda+q_{0}\right) \lambda e^{-\lambda \tau}} \\
& =-\frac{\left(3 \lambda^{2}+2 p_{2} \lambda+p_{1}\right) e^{\lambda \tau}}{\left(q_{1} \lambda+q_{0}\right) \lambda}+\frac{q_{1}}{\left(q_{1} \lambda+q_{0}\right) \lambda}-\frac{\tau}{\lambda} .
\end{aligned}
$$

Then

$$
\begin{gathered}
\operatorname{sgn}\left[\frac{d(\operatorname{Re} \lambda)}{d \tau}\right]_{\tau=\tau_{k}} \\
=\operatorname{sgn}\left[\operatorname{Re}\left(\frac{d \lambda}{d \tau}\right)^{-1}\right]_{\lambda=i \omega_{0}} \\
=\operatorname{sgn}\left[\operatorname { R e } \left(-\frac{\left(3 \lambda^{2}+2 p_{2} \lambda+p_{1}\right) e^{\lambda \tau}}{\left(q_{1} \lambda+q_{0}\right) \lambda}\right.\right. \\
\left.\left.+\frac{q_{1}}{\left(q_{1} \lambda+q_{0}\right) \lambda}-\frac{\tau}{\lambda}\right)\right] \\
=\operatorname{sgn} \operatorname{Re}\left[-\frac{\left(p_{1}-3 \omega_{0}^{2}+2 p_{2} \omega_{0} i\right)\left[\cos \left(\omega_{0} \tau_{k}\right)+i \sin \left(\omega_{0} \tau_{k}\right)\right]}{\left(q_{1} \omega_{0} i+q_{0}\right) \omega_{0} i}\right. \\
\left.+\frac{q_{1}}{\left(q_{1} \omega_{0} i+q_{0}\right) \omega_{0} i}\right]
\end{gathered}
$$




$$
\begin{aligned}
&=\operatorname{sgn} \frac{1}{\Lambda}\{ {\left[\left(p_{1}-3 \omega_{0}^{2}\right) \cos \left(\omega_{0} \tau_{k}\right)-2 p_{2} \omega_{0} \sin \left(\omega_{0} \tau_{k}\right)\right] } \\
& \times\left(q_{1} \omega_{0}^{2}\right) \\
&-\left[\left(p_{1}-3 \omega_{0}^{2}\right) \sin \left(\omega_{0} \tau_{k}\right)+2 p_{2} \omega_{0} \cos \left(\omega_{0} \tau_{k}\right)\right] \\
&\left.\times q_{0} \omega_{0}-q_{1}^{2} \omega_{0}^{2}\right\} \\
&=\operatorname{sgn} \frac{1}{\Lambda}\left\{\left(3 \omega_{0}^{2}-p_{1}\right) \omega_{0}\left[q_{1} \omega_{0} \cos \left(\omega_{0} \tau_{k}\right)-q_{0} \sin \left(\omega_{0} \tau_{k}\right)\right]\right. \\
&\left.-2 p_{2} \omega_{0}^{2}\left[q_{1} \omega_{0} \sin \left(\omega_{0} \tau_{k}\right)+q_{0} \cos \left(\omega_{0} \tau_{k}\right)\right]-q_{1}^{2} \omega_{0}^{2}\right\} \\
&=\operatorname{sgn} \frac{1}{\Lambda}\left[3 \omega_{0}^{6}+2\left(p_{2}^{2}-p_{1}\right) \omega_{0}^{4}+\left(p_{1}^{2}-2 p_{0} p_{2}-q_{1}^{2}\right) \omega_{0}^{2}\right] \\
&=\operatorname{sgn} \frac{\omega_{0}^{2}}{\Lambda}\left[3 \omega_{0}^{4}+2 D_{2} \omega_{0}^{2}+D_{1}\right] \\
&=\operatorname{sgn} \frac{\omega_{0}^{2}}{\Lambda}\left\{h^{\prime}\left(\omega_{0}^{2}\right)\right\}=\operatorname{sgn}\left\{h^{\prime}\left(\omega_{0}^{2}\right)\right\},
\end{aligned}
$$

where $\Lambda=q_{1}^{2} \omega_{0}^{4}+q_{0}^{2} \omega_{0}^{2}$. It follows from the hypothesis $\left(H_{3}\right)$ that $h^{\prime}\left(\omega_{0}^{2}\right) \neq 0$ and therefore the transversality condition holds.

Lemma 5. All the roots of (9), except a zero root, have negative real parts when $p_{1}>q_{1}$; (i) of Lemma 2 and $\tau \in\left[0, \tau_{0}\right.$ ) hold.

Proof. Consider

$$
\lambda^{3}+p_{2} \lambda^{2}+\left(p_{1}-q_{1}\right) \lambda+p_{0}-q_{0}=\lambda\left(\lambda^{2}+p_{2} \lambda+p_{1}-q_{1}\right) .
$$

It is easy to get that the roots of (23) are $\lambda_{1}=0$ and $\lambda_{2,3}=$ $\left(-p_{2} \pm \sqrt{p_{2}^{2}-4\left(p_{1}-q_{1}\right)}\right) / 2$. If $p_{1}-q_{1}>0$, all the roots of $(23)$, except a zero root, have negative real parts. We complete the proof.

Summarizing the previous discussions, we have the following result.

Theorem 6. Suppose that the conditions $\left(H_{1}\right),\left(H_{2}\right)$, and $\left(H_{3}\right)$ are satisfied.

(i) If $d=d_{0} \neq d_{1}$ and $\tau \in\left[0, \tau_{0}\right)$, then the system (1) undergoes a Pitchfork bifurcation at positive equilibrium $E^{*}$.

(ii) If $d=d_{0} \neq d_{1}$ and $\tau=\tau_{0}$, then system (1) can undergo a Hopf-Pitchfork bifurcation at the positive equilibrium $E^{*}$.

\section{Conclusions}

In this section, we present some particular cases of system (1) as follows:

$$
\begin{gathered}
\frac{d P}{d t}=r_{1} P\left(1-\frac{P}{K}\right)-a P Z, \\
\frac{d Z}{d t}=b Z P-c Z-d \frac{P(t-\tau)}{e+P(t-\tau)} Z .
\end{gathered}
$$

From [2], we know that the system (24) undergoes a Hopf bifurcation at the positive equilibrium. In this paper, we get the condition that (9) has a zero root and also get the conditions that (9) has double zero roots. Furthermore, we obtain the conditions that (9) has a single zero root and a pair of purely imaginary roots. Under this condition, system (1) undergoes a Hopf-Pitchfork bifurcation at the positive equilibrium. Especially, when $\tau=0$, system (24) reduces to

$$
\begin{gathered}
\frac{d P}{d t}=r_{1} P\left(1-\frac{P}{K}\right)-a P Z, \\
\frac{d Z}{d t}=b Z P-c Z-d \frac{P(t)}{e+P(t)} Z .
\end{gathered}
$$

We can conclude that the positive equilibrium $E\left(P^{*}, Z^{*}, W^{*}\right)$ is locally asymptotically stable in the absence of toxin delay.

\section{Acknowledgments}

The authors are grateful to the referees for their valuable comments and suggestions on the paper. The research of the authors was supported by the Fundamental Research Fund of Henan University (2012YBZR032).

\section{References}

[1] S. Khare, O. P. Misra, C. Singh, and J. Dhar, "Role of delay on planktonic ecosystem in the presence of a toxic producing phytoplankton," International Journal of Differential Equations, vol. 2011, Article ID 603183, 16 pages, 2011.

[2] J. Chattopadhyay, R. R. Sarkar, and A. El Abdllaoui, "A delay differential equation model on harmful algal blooms in the presence of toxic substances," IMA Journal of Mathematics Applied in Medicine and Biology, vol. 19, no. 2, pp. 137-161, 2002.

[3] R. R. Sarkar and J. Chattopadhayay, "Occurrence of planktonic blooms under environmental fluctuations and its possible control mechanism-mathematical models and experimental observations," Journal of Theoretical Biology, vol. 224, no. 4, pp. 501-516, 2003.

[4] S. Chakraborty, S. Roy, and J. Chattopadhyay, "Nutrient-limited toxin production and the dynamics of two phytoplankton in culture media: a mathematical model," Ecological Modelling, vol. 213, no. 2, pp. 191-201, 2008.

[5] K. Das and S. Ray, "Effect of delay on nutrient cycling in phytoplankton-zooplankton interactions in estuarine system," Ecological Modelling, vol. 215, no. 1-3, pp. 69-76, 2008.

[6] S. R.-J. Jang, J. Baglama, and J. Rick, "Nutrient-phytoplanktonzooplankton models with a toxin," Mathematical and Computer Modelling, vol. 43, no. 1-2, pp. 105-118, 2006.

[7] B. Mukhopadhyay and R. Bhattacharyya, "Modelling phytoplankton allelopathy in a nutrient-plankton model with spatial heterogeneity," Ecological Modelling, vol. 198, no. 1-2, pp. 163173, 2006. 
[8] S. Roy, "The coevolution of two phytoplankton species on a single resource: allelopathy as a pseudo-mixotrophy," Theoretical Population Biology, vol. 75, no. 1, pp. 68-75, 2009.

[9] T. Saha and M. Bandyopadhyay, "Dynamical analysis of toxin producing phytoplankton-zooplankton interactions," Nonlinear Analysis: Real World Applications, vol. 10, no. 1, pp. 314-332, 2009.

[10] J. M. Cushing, Integrodifferential Equations and Delay Models in Population Dynamics, Springer, Berlin, Germany, 1977.

[11] Y. Kuang, Delay Differential Equations with Applications in Population Dynamics, vol. 191, Academic Press, New York, NY, USA, 1993.

[12] Y.-H. Fan and L.-L. Wang, "Periodic solutions in a delayed predator-prey model with nonmonotonic functional response," Nonlinear Analysis: Real World Applications, vol. 10, no. 5, pp. 3275-3284, 2009.

[13] G.-P. Hu and W.-T. Li, "Hopf bifurcation analysis for a delayed predator-prey system with diffusion effects," Nonlinear Analysis: Real World Applications, vol. 11, no. 2, pp. 819-826, 2010.

[14] Z.-P. Ma, H.-F. Huo, and C.-Y. Liu, "Stability and Hopf bifurcation analysis on a predator-prey model with discrete and distributed delays," Nonlinear Analysis: Real World Applications, vol. 10, no. 2, pp. 1160-1172, 2009.

[15] X.-P. Yan and W.-T. Li, "Bifurcation and global periodic solutions in a delayed facultative mutualism system," Physica D, vol. 227, no. 1, pp. 51-69, 2007.

[16] Z. Zhang and Z. Wang, "Periodic solution for a two-species nonautonomous competition Lotka-Volterra patch system with time delay," Journal of Mathematical Analysis and Applications, vol. 265, no. 1, pp. 38-48, 2002.

[17] Y. Song, M. Han, and J. Wei, "Stability and Hopf bifurcation analysis on a simplified BAM neural network with delays," Physica D, vol. 200, no. 3-4, pp. 185-204, 2005. 


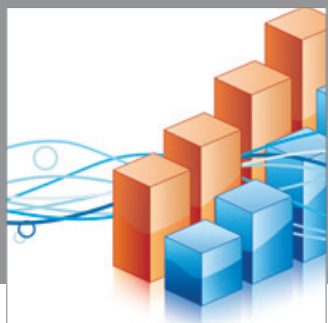

Advances in

Operations Research

mansans

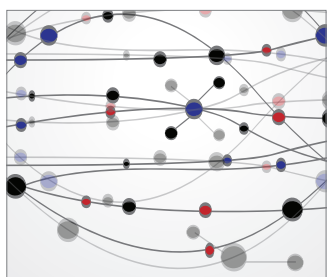

The Scientific World Journal
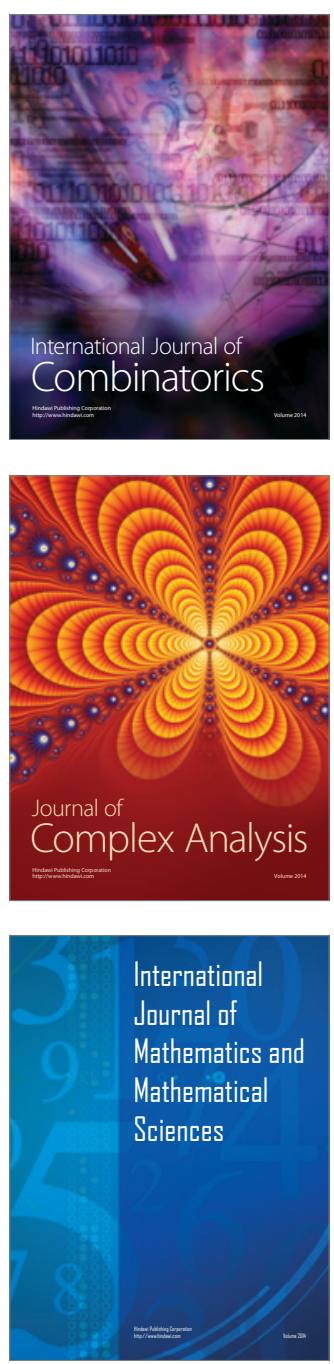
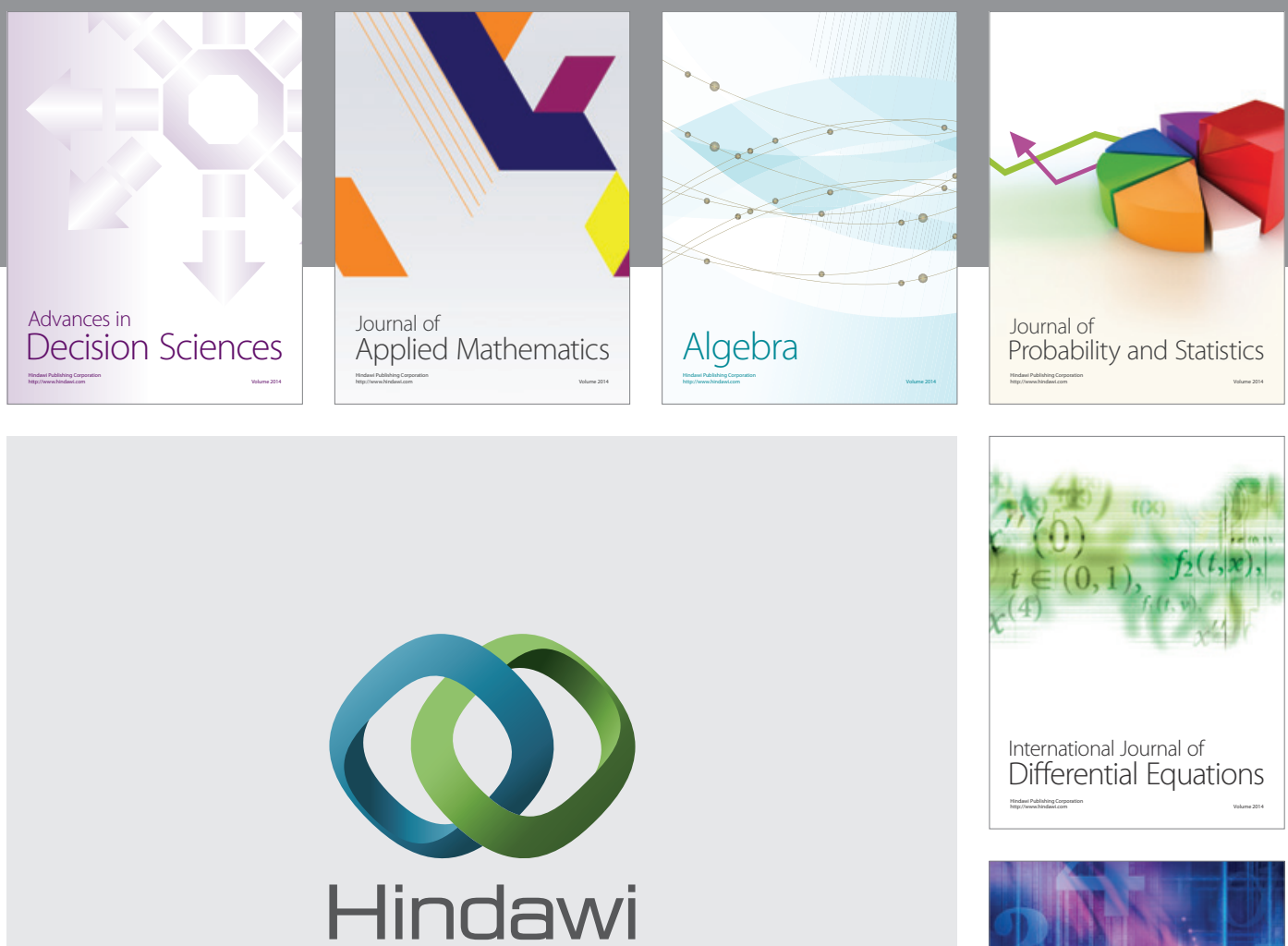

Submit your manuscripts at http://www.hindawi.com
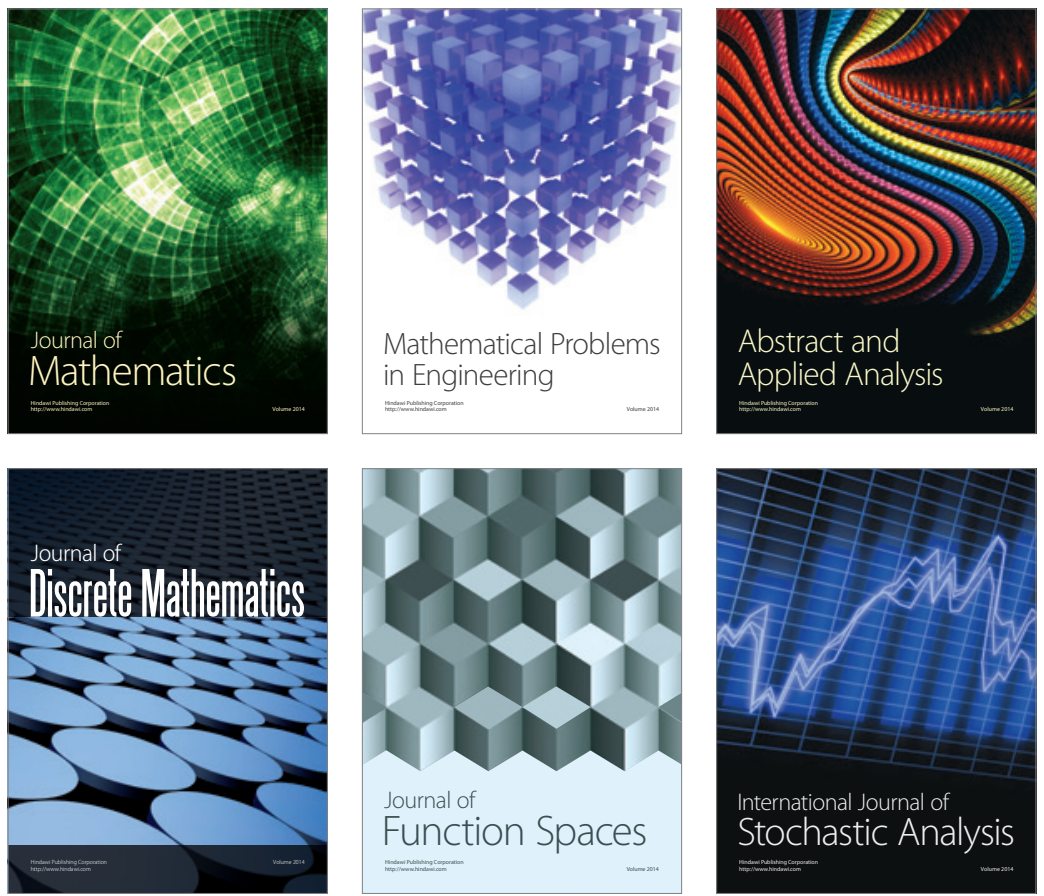

Journal of

Function Spaces

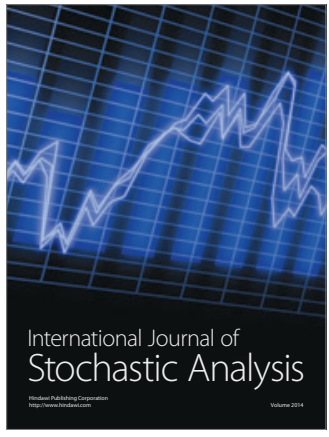

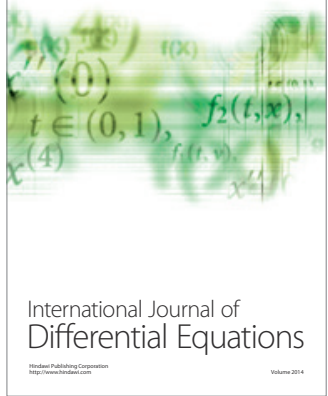
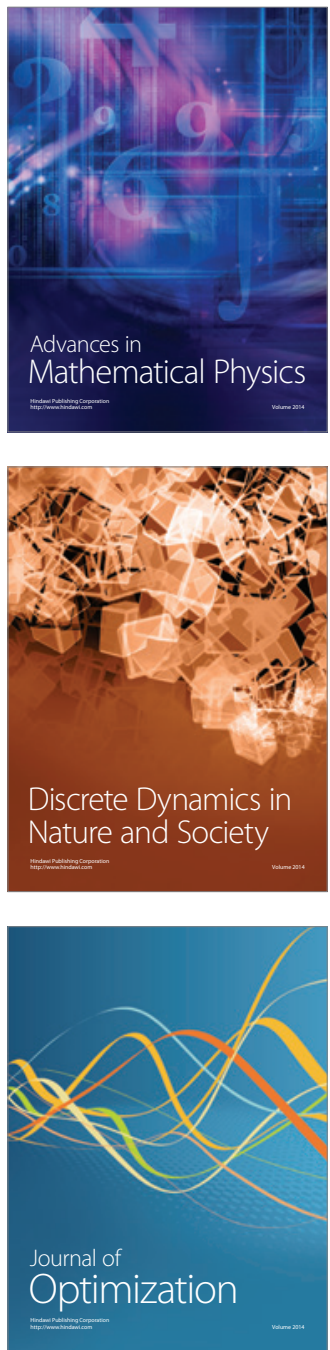